\title{
Respiratory Sinus Arrhythmia Attenuation via Targeted Lung Denervation in Sheep and Humans
}

\author{
Arschang Valipour $^{\mathrm{a}}$ Martin L. Mayse ${ }^{\mathrm{b}} \quad$ Alexander D. Peterson $^{\mathrm{b}}$ \\ Philip J. Johnson $^{b}$ Kristina T. Rouw ${ }^{b}$ Sherwin Asadi ${ }^{d}$ James P. Hummel ${ }^{c}$ \\ ${ }^{a}$ Department of Respiratory and Critical Care Medicine, Karl-Landsteiner-Institute for Lung Research and \\ Pulmonary Oncology, Krankenhaus Nord-Klinik Floridsdorf, Vienna, Austria; b Nuvaira Inc., Minneapolis, MN, USA; \\ 'Division of Cardiovascular Medicine, University of Wisconsin, Madison, WI, USA; ${ }^{d}$ Department of Pediatrics, \\ SMZO Wien, Vienna, Austria
}

\section{Keywords}

Targeted lung denervation - Respiratory sinus arrhythmia . COPD therapy · Parasympathetic

\begin{abstract}
Background: Targeted lung denervation (TLD) is a novel bronchoscopic therapy that disrupts parasympathetic pulmonary nerve input to the lung. Parasympathetic input to the heart originating from the lungs contributes to respiratory sinus arrhythmia (RSA) and disruption of pulmonary nerves via TLD may impact RSA. Objective: The aim of this study was to assess the potential of TLD to affect RSA in sheep and humans. Methods: TLD was performed in 5 sheep and 9 humans using a novel lung denervation system (Nuvaira Inc., Minneapolis, MN, USA) with an electrocardiogram collected before and after the procedure. Frequency domain analysis of heart rate variability was performed in 5 sheep and 6 humans with presence of RSA approximated as highfrequency power (HF power). Results: HF power decreased in 3 of 5 sheep with 1 animal reaching less than $7 \%$ of its baseline HF power 30 days after TLD. The average treatment location was more distal in the remaining 2 animals, which
\end{abstract}

did not exhibit RSA attenuation, suggesting diminished denervation. HF power decreased in 5 of 6 humans, with 3 subjects reaching less than $50 \%$ of their baseline HF power 90 days after TLD. Conclusions: TLD appeared to attenuate RSA in both sheep and human cohorts of this sub-study. Further confirmation in humans is necessary to allow for RSA attenuation to be used as a marker of successful lung denervation via TLD.

(c) 2019 The Author(s)

Published by S. Karger AG, Basel

\section{Introduction}

Increased cholinergic tone of parasympathetic nerves is a primary mechanism in obstructive lung disease [1]. Abundant release of acetylcholine via pulmonary nerves causes bronchoconstriction, increased mucus production, and inflammation [2]. Well established improvement in these obstructive symptoms is achieved through pharmacologic blockade of acetylcholine by inhalation of anticholinergics [3].

Targeted lung denervation (TLD) is a novel bronchoscopic therapy that disrupts parasympathetic pulmonary

Assoc. Prof. Arschang Valipour, MD

Department of Respiratory and Critical Care Medicine

Karl-Landsteiner-Institute for Lung Research and Pulmonary Oncology

Krankenhaus Nord-Klinik Floridsdorf, Brünnerstrasse 68

AT-1210 Vienna (Austria)

E-Mail arschang.valipour@wienkav.at 
nerves via radio frequency (RF) ablation. Durable reduction of parasympathetic input to the lungs has the potential to relax smooth muscle, decrease mucus production, and attenuate airway hyper-responsiveness [4-6]. Together, these improvements are expected to reduce the occurrence of exacerbations in moderate to severe COPD patients $[7,8]$. Along with the intended effect of relieving obstructive airway symptoms, attenuation of pulmonary parasympathetic activity may be evident by secondary effects on other dependent autonomic systems.

Fluctuation in heart rate beat to beat, or heart rate variability (HRV), is driven by modulation of autonomic input to the heart [9]. Cyclic variability in heart rate that follows respiration, or respiratory sinus arrhythmia (RSA), is a component of HRV attributed primarily to parasympathetic feedback [10-13]. One parasympathetic pathway modulating heart rate in response to respiration is vagal feedback from pulmonary stretch receptors [11, 13]. Disruption of pulmonary vagal nerve branches may attenuate the pulmonary stretch pathway, thereby altering RSA. The aim of this study is to explore TLD's potential effect on RSA in both sheep and humans.

\section{Methods}

\section{TLD Procedure}

Bilateral denervation is performed using a bronchoscopic lung denervation system (Nuvaira, Minneapolis, MN, USA) with fluoroscopic guidance using standard techniques. Ablations are performed circumferentially in each of the left and right mainstem bronchi and have been previously described $[8,14-16]$. The bronchial surface is protected during RF delivery by cooled fluid passing through the electrode and balloon that continues for a short time after each activation. Further details of the TLD procedure are provided in the online supplementary Table E1 (for all online suppl. material, see www.karger.com/doi/10.1159/000501623).

\section{RSA Data Analysis and Equating RSA to High-Frequency}

Power

A standard three-lead electrocardiogram (ECG) was recorded ( $1 \mathrm{kHz}$ sampling) before and after TLD as outlined in the following model-specific sections. HRV analysis was preceded by identifying and removing artifacts caused by ectopic beats to minimize potential skew of frequency domain measures. Suitable R-R interval sequences were then imported into Kubios HRV Premium 3.0.2 software for frequency domain analysis. As per Kubios guidelines, R-R interval sequences having more than $5 \%$ artifacts were not analyzed in this study. This software transformed each signal into an evenly sampled time series before applying the fast Fourier transform to estimate its respective power spectrum. Power contained within predefined frequency bands was also calculated using the Kubios software.

Presence of RSA was estimated as power contained within the high-frequency (HF) band (HF power) aiming to account for both the parasympathetic drive of HRV at this frequency and the rate at which subjects breathe. The HF band was defined as $0.15-0.4 \mathrm{~Hz}$ when analyzing human spectra $[9,10]$. A modified HF band of $0.10-0.4 \mathrm{~Hz}$ was implemented when analyzing sheep spectra to account for the lower ventilation rate. Further details of the HRV measurements and the Kubios software are provided in online supplementary Table E2.

\section{Preclinical Study}

The procedures involving the care and use of animals in this study were reviewed and approved by the test facility's Animal Care and Use Committee prior to initiation and were performed in accordance with the Animal Welfare Act of 1966. TLD was performed in 5 sheep (51-66 kg) with a 30-day follow-up. Although human treatments are performed in the mainstem bronchi, anatomy specific to the sheep lung often prevents treatment in the mainstem bronchi causing treatments to be performed more distally. Treatments distal to the mainstem bronchi leave the proximal (upstream) portion of the lung innervated. The TLD treatment location for each activation was recorded based on the number of side branching airways between the treatment site and the main carina.

An ECG was recorded for $5 \mathrm{~min}$ before and after the TLD procedure and at the 30-day follow-up. Animals were under anesthesia (isoflurane) and positive pressure ventilation during all assessments. All animals were ventilated at either 7 or 10 breaths per minute (BrPM) across assessments to maintain $\mathrm{ETCO}_{2}$.

A total of 15 ECG recordings were collected. QRS complexes were identified in 2.5-min segments of each recording and the corresponding sequences of R-R intervals were checked for artifacts. One analyzed sequence contained $<1 \%$ artifacts which were removed. The remaining 14 sequences were free of artifacts. The R-R interval sequences were then imported into Kubios software.

\section{Clinical Sub-Study}

This study was approved by the institution's ethics committee. Patients with moderate to severe COPD were recruited to participate in a study (clinical trial No. NCT01716598) investigating the feasibility of TLD therapy [16]. Patients recruited at the Otto-Wagner-Spital, Vienna, Austria were included in this sub-study investigating TLD's potential impact on RSA.

An ECG was recorded for 9 patients via a non-invasive cardiovascular monitoring system (Task Force ${ }^{\circledR}$ Monitor, CNSystems, Graz, Austria) during screening and the 90-day follow-up assessment. During recording, patients were instructed to lie supine, resting quietly for three 10 -min windows separated by 2 -min windows where the subjects were asked a series of simple questions (simple math problems and color identification). Subjects breathed in sync with a metronome at a frequency that was comfortable for them. The metronome frequency was not standardized across patients or assessments.

RSA analysis was performed on recordings for 6 of the 9 patients. Two patients were excluded because of recording errors during one of the assessments ( 1 patient at screening and 1 patient at the 90-day follow-up) that prevented analysis. The third excluded patient did not attend the 90-day follow-up. All remaining patients had recordings for both assessments. QRS complexes were identified in 5-min segments from each resting window, making three sequences of R-R intervals at screening and at the 90-day follow-up for each patient. One resting sequence at the 90-day follow-up contained $8 \%$ artifacts and was excluded from analysis. All 


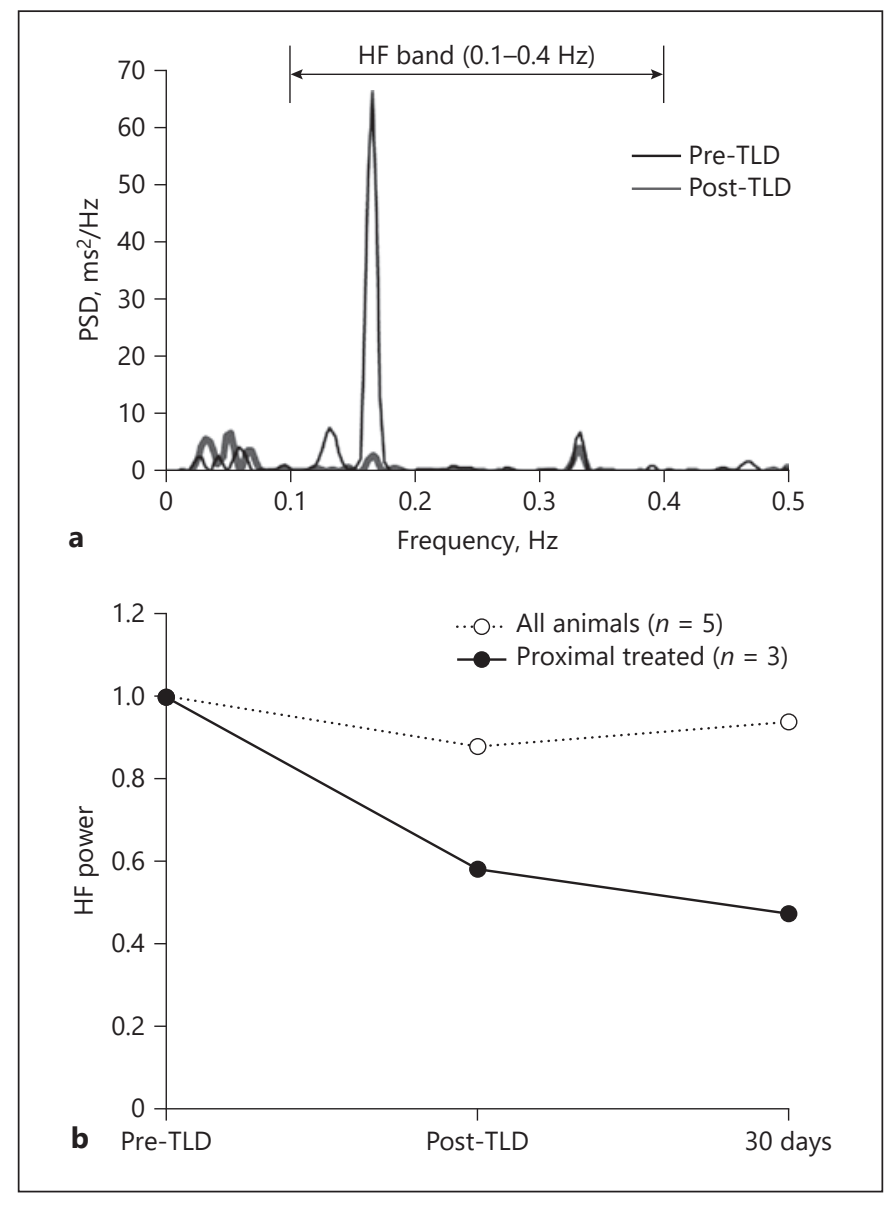

Fig. 1. Preclinical frequency domain analysis of HRV. a Estimated power spectra for animal A3 before and immediately after TLD. Power is concentrated at $0.167 \mathrm{~Hz}$ which matches the ventilation rate of $10 \mathrm{BrPM}$. HF power is substantially attenuated after TLD. b Average HF power is attenuated after TLD for proximally treated animals up to 30 days.

other recordings contained $<5 \%$ artifacts which were manually removed before importing the sequences into Kubios software. Assuming independence of resting windows per assessment, estimated power spectra and power within predefined frequency bands were averaged between the three sequences per patient per assessment, except for 1 patient with only two sequences to average at the 90-day follow-up.

\section{Results}

\section{Preclinical Study}

Power spectra were estimated for all 5 animals at three separate assessments. Before TLD, all sheep demonstrated HRV in sync with their respiration rate. Figure la shows representative power spectra estimates for an ani-

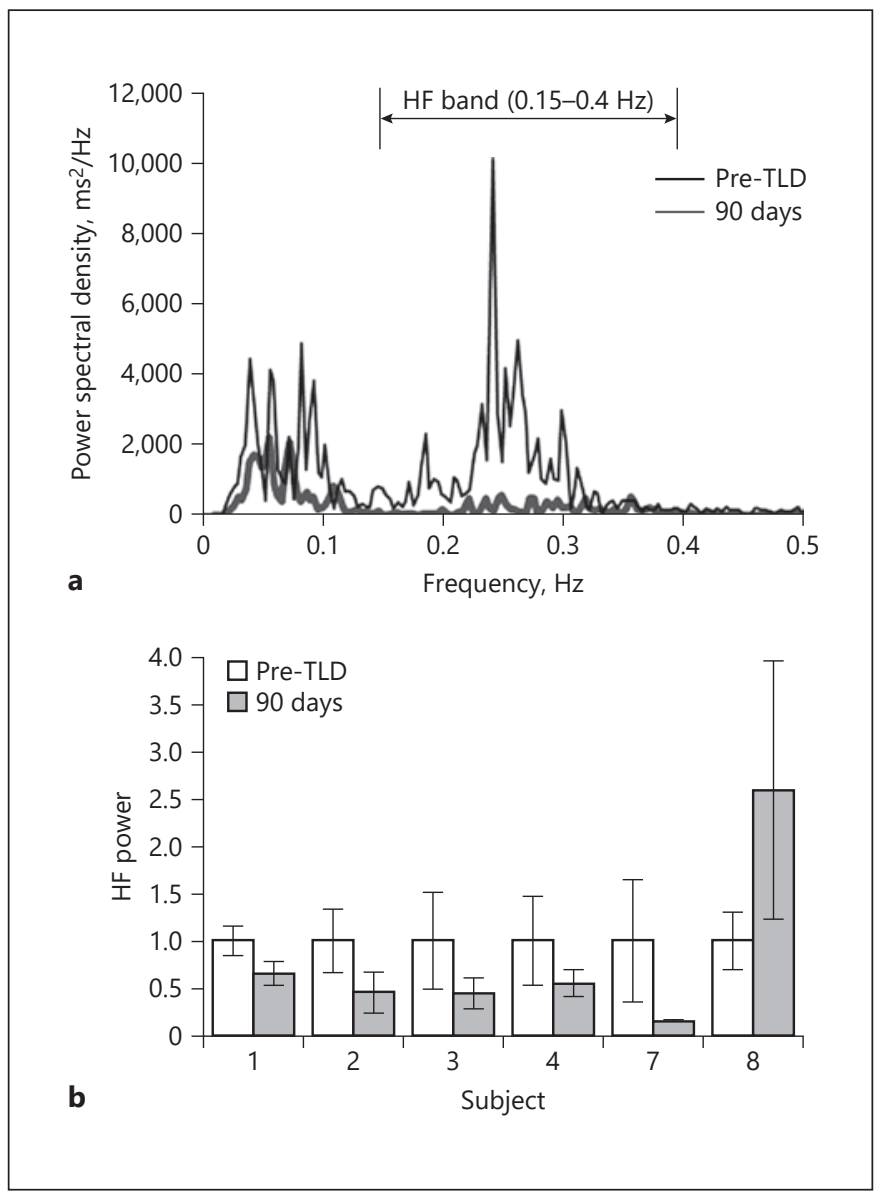

Fig. 2. Clinical frequency domain analysis of HRV. a Average estimated power spectra for Patient 7. HF power is attenuated at the 90-day follow-up. b HF power pre-TLD and at the 90-day followup. HF power is reduced in 5 of 6 patients, with patient 8 showing an increase from baseline. Error bars represent 1 standard deviation.

mal immediately before and after TLD. The data demonstrate a dramatic decrease in HRV at respiration frequency $(0.167 \mathrm{~Hz})$ when comparing pre-TLD to post-TLD, indicating immediate attenuation of RSA.

HF power per assessment was normalized to each animal's baseline and averaged across animals in Figure $1 \mathrm{~b}$. There was a minimal decrease in average HF power as only 3 of 5 sheep presented decreased HF power after TLD. One potential cause for difference in HF power between the animals is treatment location. Anatomy of the sheep lungs dictated where the electrode was placed during RF delivery, and average treatment location was inconsistent between animals. The average of eight treatment locations in each of the 2 animals without a decrease in HF power after TLD was distal (more than five airways 
beyond the main carina on average). Distal treatments do not disrupt upstream (proximal) innervation of the lung. In the remaining 3 animals who all presented decreased HF power, the average treatment location was fewer than five airways beyond the main carina. Having more proximal treatments allowed for disruption of more innervated lung segments. When excluding the two distally treated animals, average HF power decreased to $47.2 \%$ of baseline 30 days after TLD. Sheep anatomy allowing for mainstem bronchi treatments as in humans would likely further improve these decreases in HF power and attenuation of RSA.

\section{Clinical Sub-Study}

Three independent power spectra were estimated at screening and 90-day follow-up for 6 subjects. Figure 2a shows a patient's average power spectra before and after treatment. Before TLD, the subject demonstrated HRV in sync with a typical breathing rate of $15 \mathrm{BrPM}$ indicating the presence of RSA. At the 90-day follow-up, a significant attenuation in this HRV was observed.

HF power was averaged per patient and normalized to each patient's baseline for comparison in Figure 2b. Five of the 6 patients showed a decrease in HF power with 3 patients decreasing below $50 \%$ of their baseline after TLD. Subject 7 demonstrated the most dramatic attenuation with HF power decreasing to $15.5 \%$ of his or her baseline. HF power increased in 1 subject at the 90-day follow-up.

\section{Discussion}

TLD is a therapy intended to disrupt parasympathetic input to the lung by ablating the peribronchial branches of the vagus nerve, in order to reduce the clinical consequences of neural hyperactivity. As the nerves targeted by TLD are also thought to play a major role in the RSA mechanism, effective denervation by TLD may attenuate RSA.

\section{Mechanism of RSA}

Fluctuations in the tone of efferent vagal motor neurons to the heart result in accelerations and decelerations of heart rate during the respiratory cycle. The afferent limb linking cyclical cardiac oscillations to the respiratory cycle remains controversial and may involve several mechanisms. It is likely that the dominant mechanism may vary among species and depend on factors such as whether breathing is spontaneous versus controlled by positive pressure, depth of anesthesia, and magnitude of pressure used for ventilation.

Respiratory Sinus Arrhythmia

Attenuation
Ludwig first observed an increase in heart rate and decrease in blood pressure during inspiration in spontaneously breathing dogs in 1847 [9]. Since then, multiple studies have linked the acceleration of heart rate on inspiration to pulmonary neural feedback. When using moderate pressures $(<15 \mathrm{~mm} \mathrm{Hg})$, inspiration induced by either positive tracheal pressure or negative intrathoracic pressure resulted in increases in heart rate. Furthermore, this reflex could be abolished with heavy anesthesia or sectioning of the cervical vagus nerve [17]. In awake spontaneously breathing animals, a dominant mechanism of RSA appears to be initiated by lung inflation. Inhibitory projections to the cardiac vagal motor neurons in the nucleus ambiguous and dorsal motor nucleus result in decreased vagal efferent tone to the heart (and thus acceleration of heart rate) during inspiration $[17,18]$.

Several additional mechanisms independent of the pulmonary vagus may also link the cardiac and respiratory cycles. Central linkage of respiratory rhythm-generating neurons with cardiovagal motor neurons may occur in the medulla [19]. In addition, the mechanical effect of changes in intrathoracic pressure during the respiratory cycle may also link the cardiac and respiratory cycles. In the non-ventilated patient, right atrial filling increases and systemic blood pressure decreases on inspiration, changes which both cause reflex increases in heart rate. It is worth noting that these mechanical influences would be reversed during positive pressure ventilation.

However, an evaluation of patients after heart and lung transplants suggests that vagal feedback from the lungs has a primary role in mediating RSA in humans [13]. The RSA present in normal controls was nearly obliterated in denervated lung transplant patients during either spontaneous or positive pressure ventilation. A small but measurable amount of residual RSA in heart transplant patients suggests that other factors including an intracardiac mechanism related to atrial stretch may play a lesser role.

\section{Clinical Relevance of RSA Attenuation via TLD}

Further characterization of the connection between TLD and RSA attenuation could allow its use as a tool for estimating the extent of peribronchial denervation achieved in a patient via TLD. RSA could be evaluated during a patient's screening visit and then re-evaluated at a follow-up visit soon after TLD. Patients without RSA attenuation could be candidates for a second TLD procedure. With reduction in RSA apparent immediately after TLD in some sheep, it is also possible that the extent of denervation could be estimated during the initial TLD 
procedure. Collection of heart rate data could be initiated just before and continue throughout the TLD procedure. Continuous assessment of RSA may allow for confirmation of denervation. Without sufficient attenuation of RSA, additional applications of RF energy could be applied during this initial procedure to enhance what appears to be incomplete denervation.

Alternatively, successful TLD may also be confirmed through abolishment of the Hering-Breuer reflex rather than changes in RSA. The Hering-Breuer reflex results from slowly adapting pulmonary stretch receptors that respond to changes in lung volume, and when activated by sustained lung inflation reflexively inhibit the onset of the subsequent inspiration. In fact, in a sub-study of a recent publication by Hummel et al. [14], the authors investigated the effects of a circumferential TLD procedure on the Hering-Breuer reflex prior to, acutely, and 7 days following the intervention. Following TLD, in the denervated sections of the lungs, over pressurization of the lung failed to alter normal breathing patterns, whereas control regions of the lung maintained the Hering-Breuer reflex. Given the complexity of physiological measurements required to demonstrate this reflex mechanism in humans in vivo, we do not believe the clinical value of its applicability in real-life practice.

Although TLD is assumed to disrupt both afferent and efferent pulmonary pathways, a recent study suggests pulmonary vagal afferent pathways regenerate while efferent pathways do not [20]. Regeneration of afferent pathways was observed by return of pulmonary stretch receptor discharge in rats 6 months after transection and re-anastomosis of the cardiopulmonary vagus nerve in that report. Pulmonary afferent regeneration after TLD in humans could potentially be observed as recovery of RSA, which in turn may serve as a physiological marker of TLD's durability on pulmonary afferent fibers. Return of RSA would not inform on the durability of efferent fiber disruption by TLD.

In addition to serving as a marker of vagal tone, several studies have suggested that RSA may serve a physiological purpose. An increase in heart rate during inspiration has been proposed to potentially reduce demand on the cardiopulmonary system by suppressing unnecessary heartbeats [21], improving ratios of anatomical dead space and cardiopulmonary shunt [22], and increasing ventilatory efficiency [11]. However, while higher RSA has been associated with improved cardiopulmonary efficiency, it remains unclear whether the oscillations in heart rate throughout the respiratory cycle are simply a marker for healthy individuals with intact vagal reflexes or actually cause significant benefit. Thus, although the loss of RSA with TLD could possibly lead to less effective cardiorespiratory coupling, any negative effects are unlikely to be clinically significant.

\section{Limitations}

The primary limitations of this study include the use of an animal model and the small sample size. Although sheep demonstrated presence of RSA before TLD, the magnitude was much lower than that of the human subjects in this study. This difference could be attributed to physiological differences between species, or the combination of anesthesia and positive pressure ventilation utilized during data collection for the sheep. Furthermore, as energy is delivered more distally in sheep due to anatomical differences, some proximal airways are left with intact nerves likely leading to less impact on RSA for sheep than humans. Attenuation of RSA in the sheep model would likely be enhanced by more proximal treatments.

Although analyzing RSA in humans during relaxed breathing was an ideal model for investigating TLD's impact on RSA, this analysis was done on a limited number of patients. Having more patients demonstrate decreased HF power after TLD would reduce the effect of patient variability. A final consideration involves the alternative mechanisms contributing to HRV that are not affected by TLD. Complete denervation of a patient's lungs via TLD may not entirely eliminate RSA, as alternative mechanisms like intracardiac stretch, baroreceptor activity, and centrally mediated rhythm may continue to bypass the affected pulmonary afferents.

\section{Conclusion}

TLD aims to ablate pulmonary nerves to reduce the clinical consequences of neural hyperactivity in patients with obstructive lung disease. This preliminary study aimed to assess the feasibility of TLD's impact on RSA by evaluating RSA before and after TLD in both sheep and humans. From these preliminary results, most of the animal and human subjects had marked attenuation of RSA after TLD. The animals which did not have reduction in the HF component of HRV were ablated more distally, leaving incomplete denervation in multiple lung segments. This suggests that measurement of RSA pre- and post-TLD may serve as a marker for the extent of denervation. A larger study is warranted to further demonstrate ablation of the pulmonary vagal branches and confirm consistent attenuation of RSA. By estimating a patient's 
RSA as the amount of HF variability in their beat to beat intervals, RSA attenuation may be an effective evaluation tool for TLD.

\section{Acknowledgements}

We would like to thank Dr. Martin Mayse, the inventor who conceived TLD therapy investigated in this study, the volunteer study participants, and the entire IPS-II research team for their work on this trial.

\section{Statement of Ethics}

Subjects who participated in the clinical trial gave their informed consent to participate in this study. The study protocol was approved by the research institute's committee on human research (clinical trial No. NCT01716598). The preclinical study was approved by the American Preclinical Services Institutional Animal Care and Use Committee (USDA registration number 41-R0074).

\section{Disclosure Statement}

All expenses were reimbursed by the study sponsor (Nuvaira Inc.). A.V. is the principal investigator of the clinical study. Both A.V. and S.A. have no conflicts of interest to disclose. J.P.H. is a paid consultant of Nuvaira and a member of their scientific advisory board. All other authors are employees at Nuvaira.

\section{Funding Sources}

The clinical trial and preclinical study were both funded by $\mathrm{Nu}$ vaira Inc.

\section{Author Contributions}

Protocol development was shared by A.V., J.P.H., and A.D.P. A.V. was involved in patient recruitment and S.A. helped A.V. with clinical data collection. Preclinical data were collected by M.L.M., A.D.P., P.J.J., and K.T.R. All authors participated in interpretation of the data and revision of the final manuscript.

\section{References}

1 Belmonte KE. Cholinergic pathways in the lungs and anticholinergic therapy for chronic obstructive pulmonary disease. Proc Am Thorac Soc. 2005;2(4):297-304.

2 Gosens R, Zaagsma J, Meurs H, Halayko AJ. Muscarinic receptor signaling in the pathophysiology of asthma and COPD. Respir Res. 2006 May;7(1):73.

3 Tashkin DP, Celli B, Senn S, Burkhart D, Kesten S, Menjoge S, et al.; UPLIFT Study Investigators. A 4-year trial of tiotropium in chronic obstructive pulmonary disease. N Engl J Med. 2008 Oct;359(15):1543-54.

4 Canning BJ. Reflex regulation of airway smooth muscle tone. J Appl Physiol (1985). 2006 Sep;101(3):971-85.

5 Ueki I, German VF, Nadel JA. Micropipette measurement of airway submucosal gland secretion. Autonomic effects. Am Rev Respir Dis. 1980 Feb;121(2):351-7.

6 McAlexander MA, Gavett SH, Kollarik M, Undem BJ. Vagotomy reverses established allergen-induced airway hyperreactivity to methacholine in the mouse. Respir Physiol Neurobiol. 2015 Jul;212-214:20-4.

7 Vogelmeier C, Hederer B, Glaab T, Schmidt H, Rutten-van Mölken MP, Beeh KM, et al.; POET-COPD Investigators. Tiotropium versus salmeterol for the prevention of exacerbations of COPD. N Engl J Med. 2011 Mar; 364(12):1093-103.

8 Valipour A, Shah PL, Pison C, Ninane V, Janssens W, Kessler R, Deslee G, Garner J, Abele C, Hartman J, Slebos DJ: Safety and dose study of targeted lung denervation in moderate/severe COPD patients. Respiration. 2019. doi: 10.1159/000500463.

9 Billman GE. Heart rate variability - a historical perspective. Front Physiol. 2011 Nov;2:86.

10 Malik M, Bigger JT, Camm AJ, Kleiger RE, Malliani A, Moss AJ, et al.; Task Force of the European Society of Cardiology and the North American Society of Pacing and Electrophysiology. Heart rate variability. Standards of measurement, physiological interpretation, and clinical use. Eur Heart J. 1996 Mar;17(3):354-81.

11 Giardino ND, Glenny RW, Borson S, Chan L. Respiratory sinus arrhythmia is associated with efficiency of pulmonary gas exchange in healthy humans. Am J Physiol Heart Circ Physiol. 2003 May;284(5):H1585-91.

12 Pumprla J, Howorka K, Groves D, Chester M, Nolan J. Functional assessment of heart rate variability: physiological basis and practical applications. Int J Cardiol. 2002 Jul;84(1):114.

13 Taha BH, Simon PM, Dempsey JA, Skatrud JB, Iber C. Respiratory sinus arrhythmia in humans: an obligatory role for vagal feedback from the lungs. J Appl Physiol (1985). 1995 Feb;78(2):638-45.

14 Hummel JP, Mayse ML, Dimmer S, Johnson PJ. Physiologic and histopathologic effects of targeted lung denervation in an animal model. J Appl Physiol (1985). 2019 Jan;126(1):6776.

15 Slebos DJ, Klooster K, Koegelenberg CF, Theron J, Styen D, Valipour A, et al. Targeted lung denervation for moderate to severe
COPD: a pilot study. Thorax. 2015 May;70(5): 411-9.

16 Valipour A, Asadi S, Pison C, Jondot M, Kessler R, Benneddif K, et al. Long-term safety of bilateral targeted lung denervation in patients with COPD. Int J Chron Obstruct Pulmon Dis. 2018 Jul;13:2163-72.

17 Anrep GV, Pascua W, Rossler R. Respiratory variations in the heart rate I. The reflex mechanism of respiratory arrhythmia. Proc R Soc Lond B Biol Sci. 1936;119(813):191-217.

18 Taylor EW, Jordan D, Coote JH. Central control of the cardiovascular and respiratory systems and their interactions in vertebrates. Physiol Rev. 1999 Jul;79(3):855-916.

19 Anrep GV, Pascua W, Rossler R. Respiratory variations in the heart rate II. The central mechanism of respiratory arrhythmia and the inter-relationship between central and reflex mechanisms. Proc R Soc Lond B Biol Sci. 1936;119(813):218-30.

20 Bregeon F, Alliez JR, Héry G, Marqueste T, Ravailhe S, Jammes Y. Motor and sensory reinnervation of the lung and heart after reanastomosis of the cervical vagus nerve in rats. J Physiol. 2007 Jun;581(Pt 3):1333-40.

21 Yasuma F, Hayano J. Respiratory sinus arrhythmia: why does the heartbeat synchronize with respiratory rhythm? Chest. 2004 Feb;125(2):683-90

22 Hayano J, Yasuma F, Okada A, Mukai S, Fujinami T. Respiratory sinus arrhythmia. A phenomenon improving pulmonary gas exchange and circulatory efficiency. Circulation. 1996 Aug;94(4):842-7. 